\title{
水中を動く網地が受ける水の抵抗一V $\mathrm{V}$. 網地の吹加れ
}

\author{
野村正恒 - 秝 敬四郎 \\ (東海区水碃研·究所).
}

\author{
Resistance of Plane Net against Flow of Water-V. \\ On the Inclination of Net in a Current. \\ Masatsune Nomura and Keishiro Mori
}

Fishing nets, when set under a current of waters, are liable to deformation. Since the synthetic fber came in use for fishing nets, the more interests have been paid for decreasing the deformation of not due to currents so as to enhance the fishing efficiency.

In ccntinuation, to the previous paper ${ }^{1}$, experiments have been carried out to clarify the different inclinations of nets constructed of various kinds of fibers, the materials of which are listed in Table 1. Using a nec stretched to a frame with an area of one square meter to make the mesh square, cne side of it was kept on the water level, and the vertical distance $h$ was measured at a current speed $v$ in a way shown in Fig. 1. Next, the inclination of the frame without the net was also determined in the same way.

Then, the inclination of the net alone was calculated by deduction this inclinate effect of the frame itself from the former one.

Thus, the relations between the inclination $h$ and the current speed $v$ were obtained as shown in Fig. 2 according to different materials of the nets.

From the calculation, the weight of the net per unit in water $w$, under water speed $v$ and angle of inclination $\theta$, is expressed on the bases of diagrams in Fig. 3 . by a formula :

$$
w=a \cdot \frac{d}{l} \cdot \tan \theta \cdot v^{2}
$$

where, $d$ is the diameter of thread, $l$ the size of a mesh ard $d$ the coefficient of resistance. It is noticed that the rets which are made of endless synthetic fibers such as Amilan (Polyamid), Saran (Vinylidere chloride), Kurehalon (Vinylidene chloride), Tevilon (Vinyle chloride) and Silklon (Polyvinyle alcohol) are larger in the value of $a$ than the nets of natural cut-fibers. In other words, $a$ is the coefficient chiefly depending upon roughness of the surface of the netting cord. In order tc decrease the inchination of nets, it is desirable to make the value of $w$ as large as possible. $a \cdot\left(\frac{d}{l}\right)$

\section{I. 緒言}

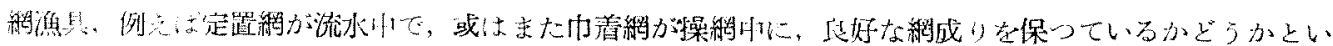

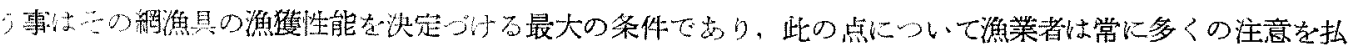

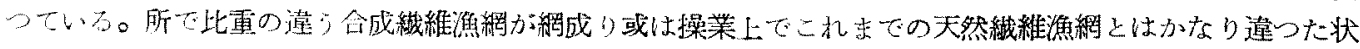

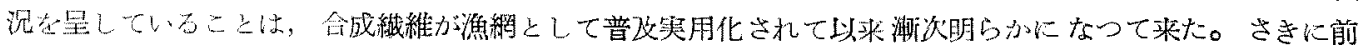

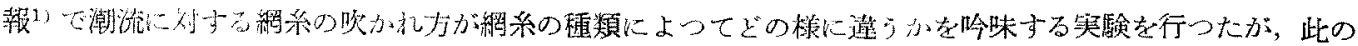

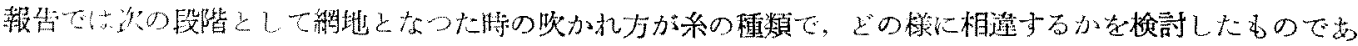
xo

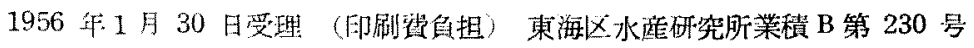




\section{II. 実験村料並びに実験方法}

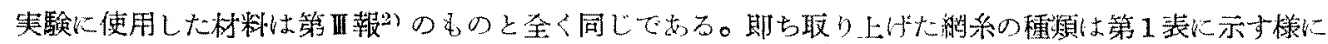

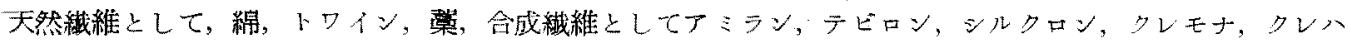
ロン，サシンの計 9 種類でるる。

Table. 1. Specifications of materials used for the experiments.

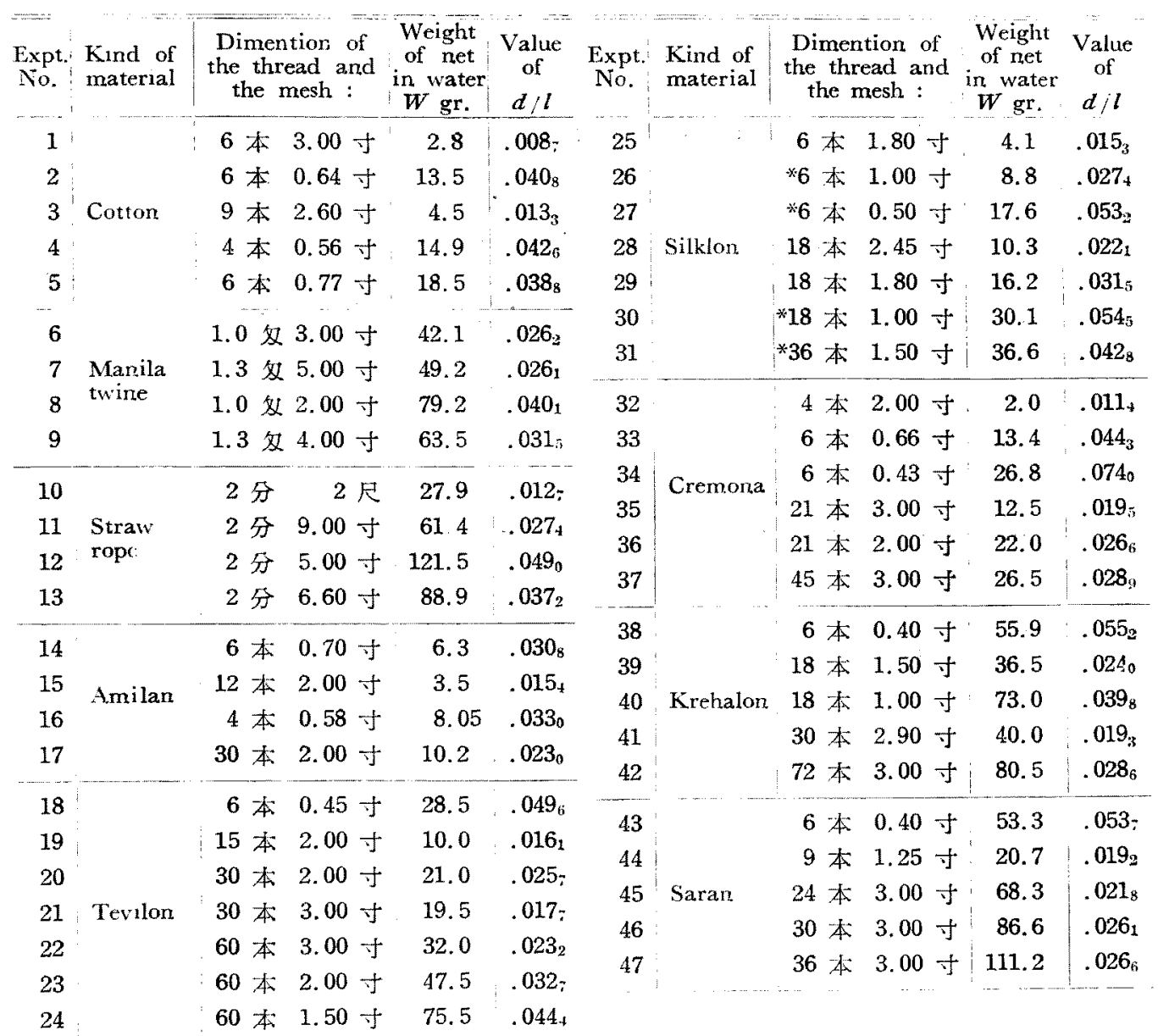

* The nets with asterisk had flat knot and all the others were of trawler knots.

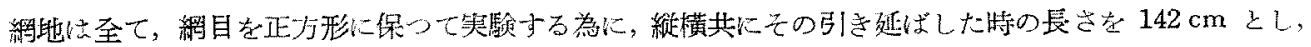

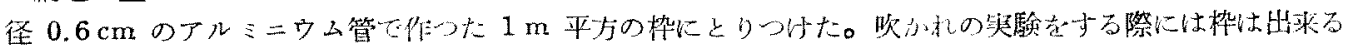

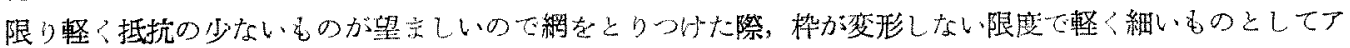
ルミニウム管を使用したむのでちる。このアルミニウム枠は水中重量が $51.5 \mathrm{~g}$ 改び $49 \mathrm{~g}$ の2つを使用し

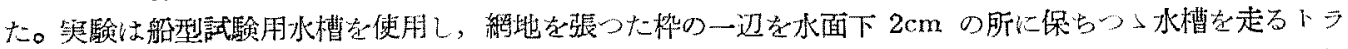
ックと共に動?様にし，他辺は自由に垂下させた。トラックを走らせて，網地に流水が当る蝫は，上辺起軸

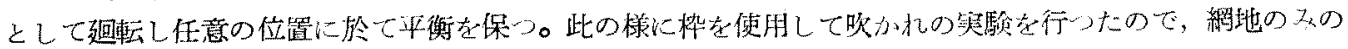

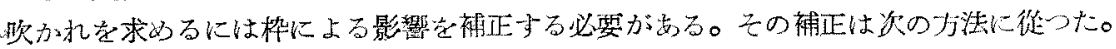

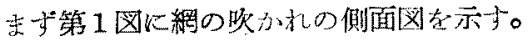

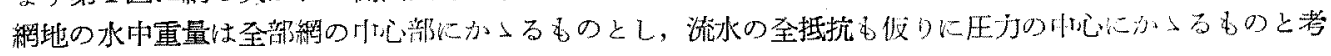




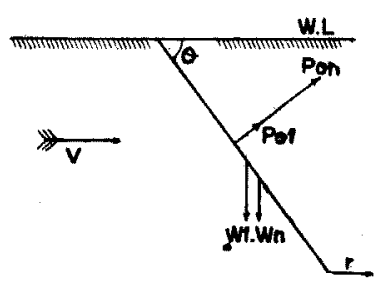

Fig. 1. Forces acting on

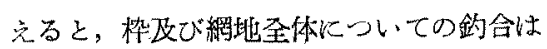

$$
P_{\theta f}+P_{\theta n(v)}=\left(W_{n}+W_{f}\right) \cos \theta-r \sin \theta \cdots \cdots(1)
$$

一う梳無して網地のみが吹かれたとした場合，欥かれの角を上と同じ $\theta$ にとる㭙はとの流速を $v^{\prime}$ とるる

$$
P_{\theta: 1\left(v^{\prime}\right)}=W_{n} \cos \theta \quad \cdots \cdots \cdots \cdots \cdots(2)
$$

又枠の欢の吹かれの鈎合は

$$
P_{\theta 1}=W_{f} \cos \theta-r \sin \theta
$$

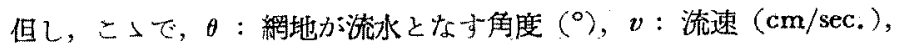
$P_{\theta n(v)}: v$ の恃の網地の全抵抗 $(\mathrm{gr}),. P_{\theta r}$ : 枠の綻棒 2 本分の抵抗 $(\mathrm{gr}$.$) ,$

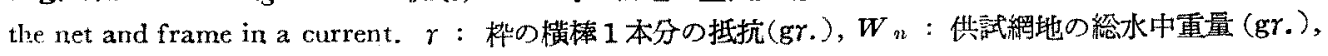
$W_{l}$ : 枠棒 3 本分の水中重量（gr）。

$$
(1) \div(2)+3.5
$$

$$
P_{\theta n(v)} / P_{\theta u\left(v^{\prime}\right)}=\left\{\left(W_{n}+W_{\gamma}\right) \cos \theta-\gamma \sin \theta-P_{\theta r}\right\} / W_{n} \cos \theta \cdots \cdots \cdots \cdots \cdots(4)
$$

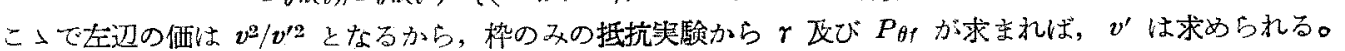
$r$ は备速度に於叮尚 $\theta=90^{\circ}$ の㭙の枠の抵抗を求めその $1 / 4$ の価をとる。一方 $P_{\theta f}$ は

$$
P_{\theta f}=\frac{\rho}{2} \cdot c_{x} \cdot d \cdot s \cdot v^{2}
$$

と扰くと, (3) 力 5

$$
{ }_{2}^{\rho} c_{x} \cdot d \cdot s \cdot v^{2}=W_{1} \cos \theta-r \sin \theta
$$

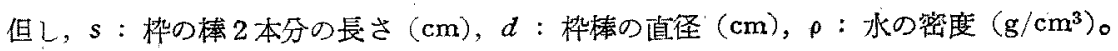

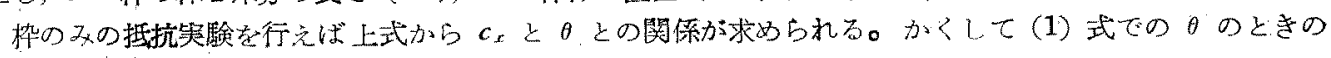
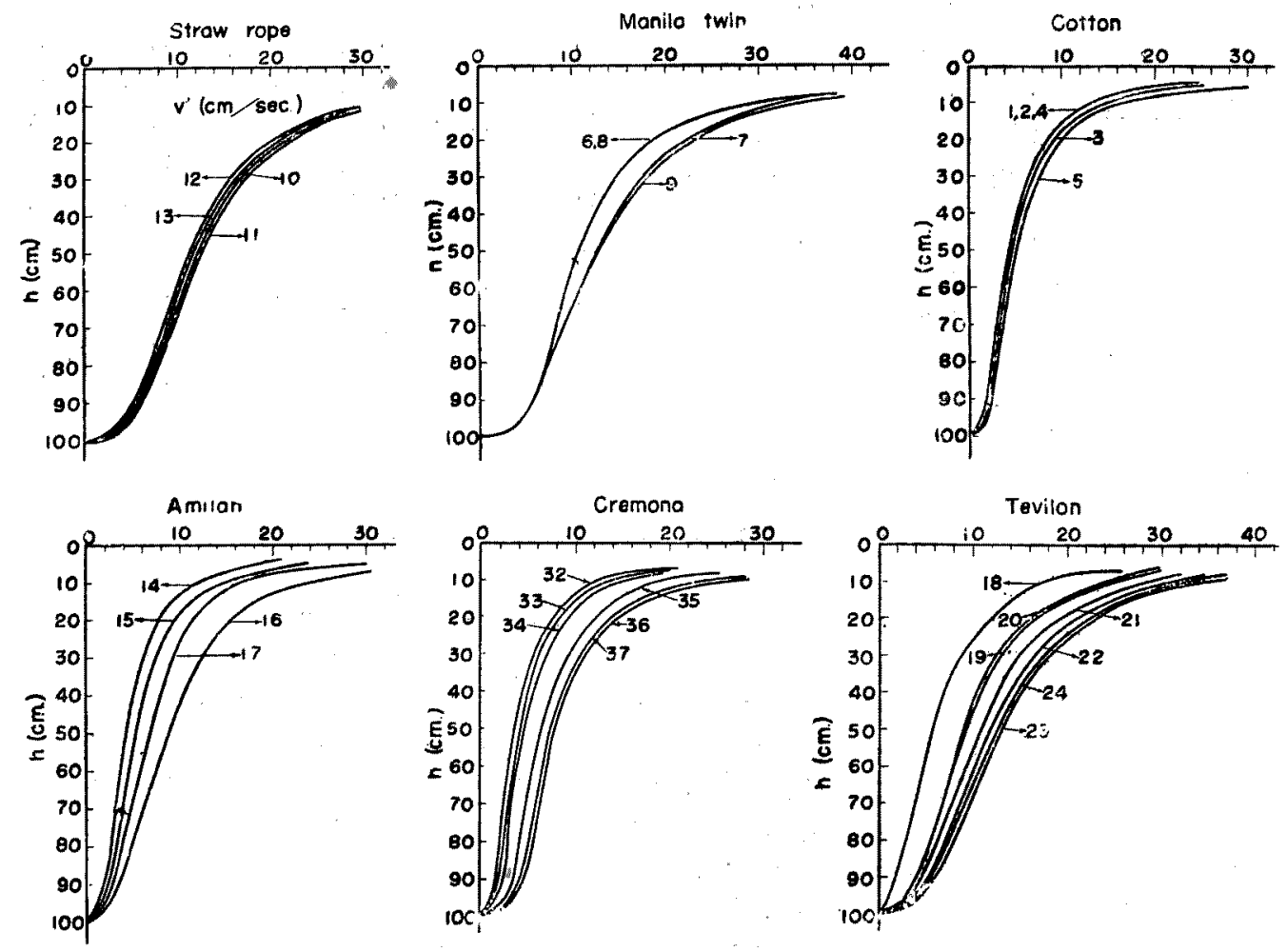

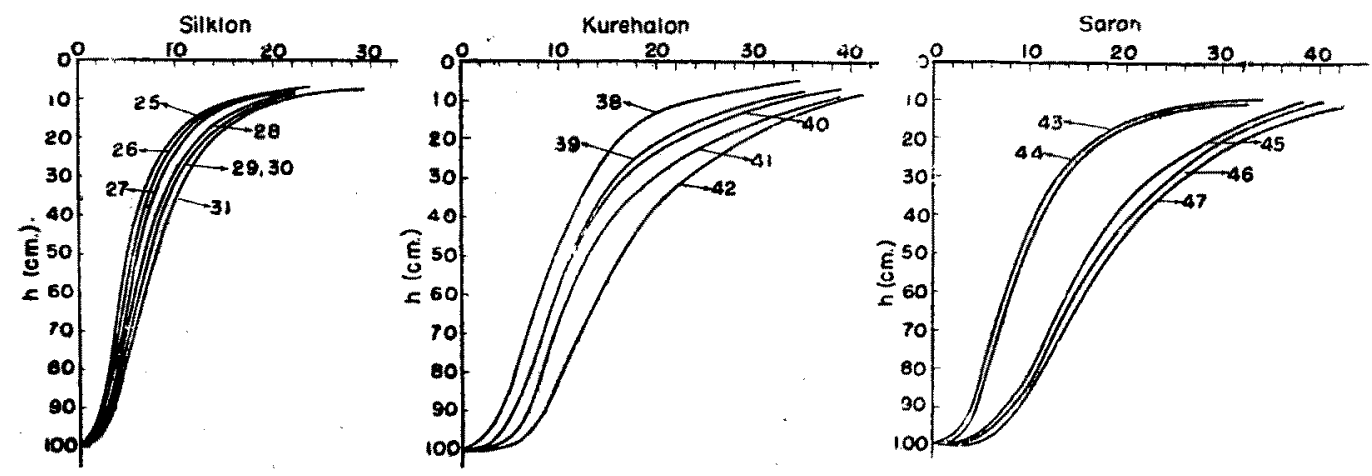

Fig. 2, The relation between current speed $v^{\prime}$ and the distance $h_{1}$ which indicate the inclination of the net.

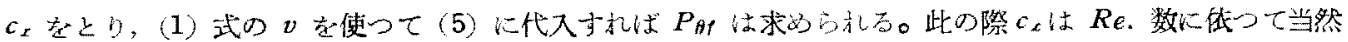

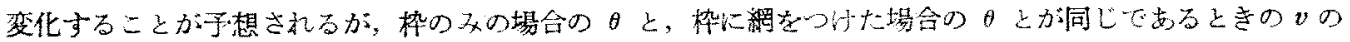

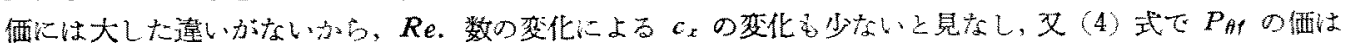

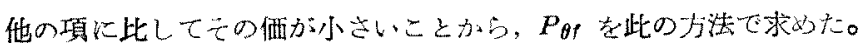

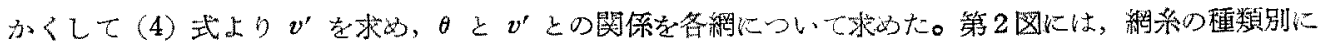

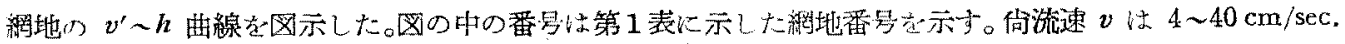

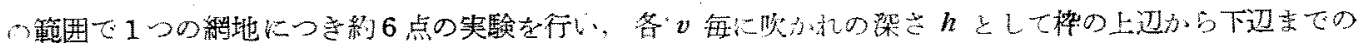
垂直距離（cm.）を測定したるのでる。

\section{III. 実験結果とその検討}

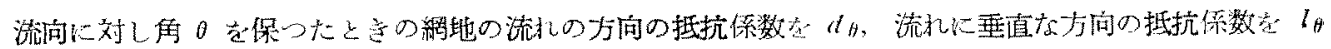

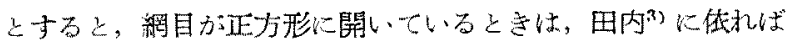

$$
\begin{gathered}
d_{\theta}=a^{\prime}\left(\begin{array}{c}
d \\
l
\end{array}\right)\left(2-\cos ^{2} \theta\right)+b^{\prime}\left(\begin{array}{l}
d \\
l
\end{array}\right)^{2} F \\
l_{\theta}=a^{\prime}\left(\begin{array}{l}
d \\
l
\end{array}\right) \sin \theta \cdot \cos \theta+b^{\prime}\left(\begin{array}{l}
d \\
l
\end{array}\right)^{2} F^{\prime}
\end{gathered}
$$

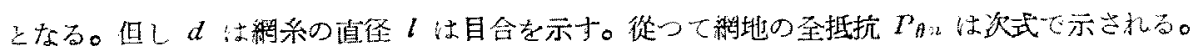

$$
p_{\theta: 2}=\left\{2 a^{\prime}\left(\begin{array}{c}
d \\
l
\end{array}\right) \sin \theta+b^{\prime}\left(\begin{array}{l}
d \\
l
\end{array}\right)^{2}\left(F \sin \theta+F^{\prime} \cos \theta\right)\right\} s \cdot v^{2}
$$

但し $s$ 網地の面積とする。

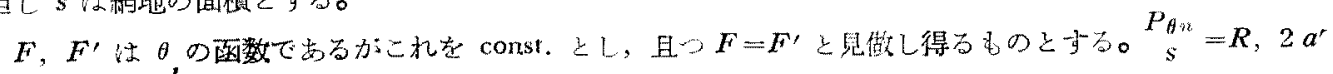
$=a, b^{\prime} F=b, \quad l=m$ 之扰々之上式保

$$
R=\left\{a m \sin \theta+b m^{2}(\sin \theta+\cos \theta)\right\} \eta^{2}
$$

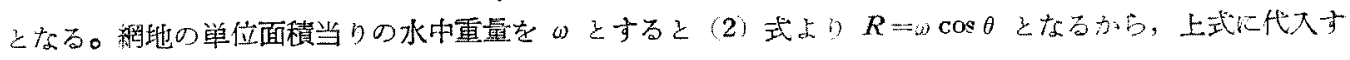
ると

$$
(a+b m) \tan \theta+b m=\frac{\omega}{m v^{2}}
$$

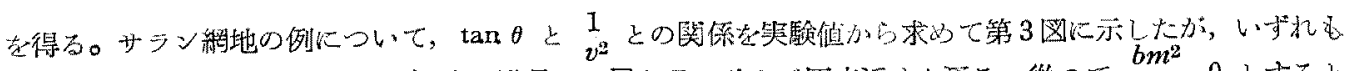

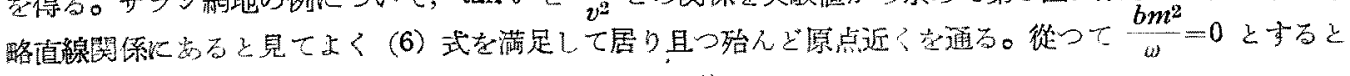
(6) 式上り

$$
\begin{aligned}
\tan \theta & =\frac{\omega}{m(a+b m) \cdot v^{2}} \\
& =\cdots \cdot \cdots \cdot v^{2}\left(1+\begin{array}{c}
b m \\
a
\end{array}\right)
\end{aligned}
$$




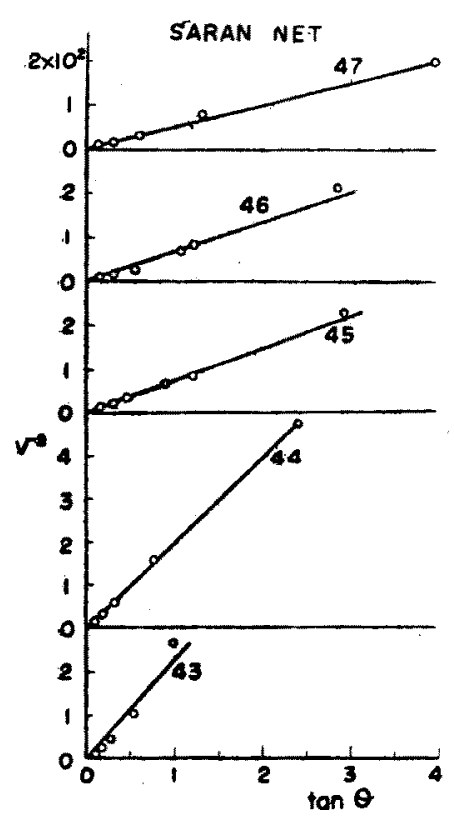

Fig. 3. The relation between value of $1 / v^{2}$ and $\tan \theta$ in case of Saran net.

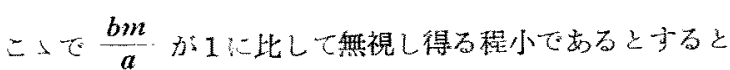

$$
\tan \theta \div \frac{\omega}{a \cdot m \cdot v^{2}}
$$

E7c30

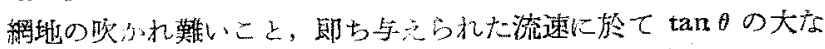
こことに対しての条件は $a$ の值の小なること並びに ${ }_{m}$ 即ち $d / l$ の值の大なることでる。

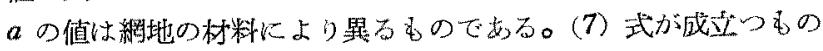

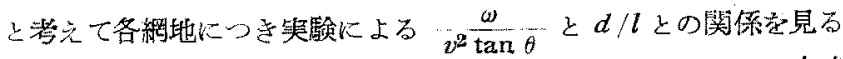

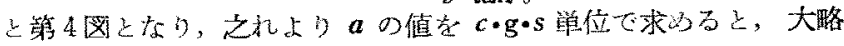

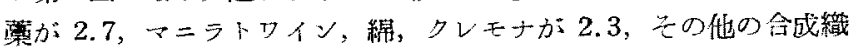
維力: 1.8 と存つた。

同一村料の糸で作られた絧地については吹かれの大小は $\frac{\omega}{d / l}$ の

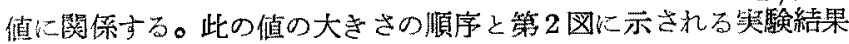

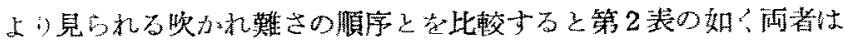
殆んど一致している。

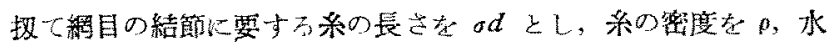
の密度支 $P_{w}$ とすると

$$
\begin{aligned}
& \omega=(2 l+\sigma d) \frac{\pi}{4} d^{2} \times\left(\rho-\rho_{w}\right)=\frac{\pi}{4}\left(\rho-\rho_{w}\right) m^{2}(2+\sigma m) l \\
& =k m^{2}(2+\sigma m) l
\end{aligned}
$$

但し $k=\frac{\pi}{4}\left(p-\rho_{w}\right) \quad \leq-5 。$
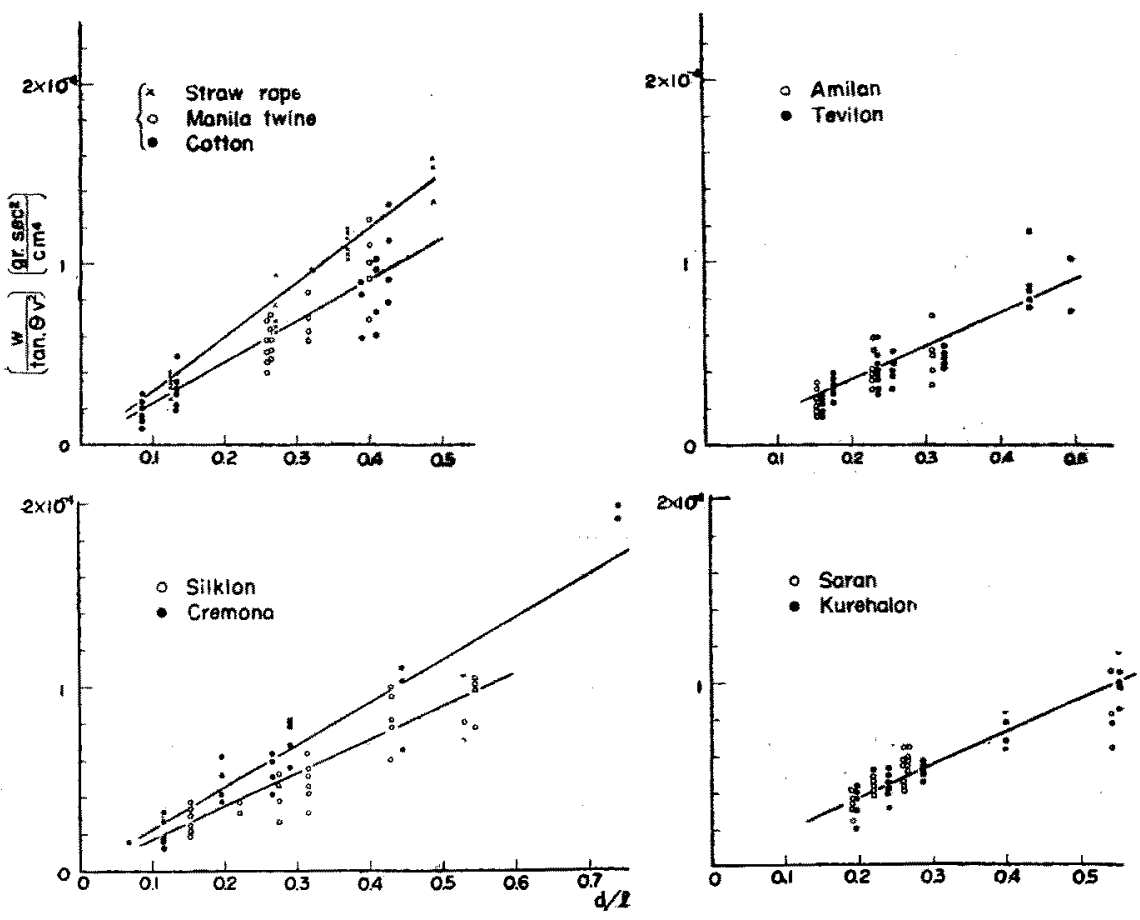

Fig. 4. The relation between value of $\frac{\omega}{\tan \theta \cdot v^{2}}$ and $\frac{d}{l}$. 
Table 2.

\begin{tabular}{|c|c|c|c|c|c|c|c|c|c|}
\hline $\begin{array}{l}\text { Expt. } \\
\text { No. }\end{array}$ & $\begin{array}{l}\text { Kind of } \\
\text { material }\end{array}$ & $\begin{array}{r}\omega \\
(\mathrm{gr} . / \mathrm{c}\end{array}$ & & $d$ & & $\stackrel{\omega}{d}$ & & $\begin{array}{l}\text { The orders } \\
\text { in value of } \frac{\omega}{d / l} \\
\text { within } \\
\text { same material }\end{array}$ & $\begin{array}{l}\text { The orders in } \\
\text { inclination } \\
\text { obtained from } \\
\text { the experiment }\end{array}$ \\
\hline $\begin{array}{l}1 \\
2 \\
3 \\
4 \\
5\end{array}$ & cotton & $\begin{array}{r}2.8 \times \\
13.5 \\
4.5 \\
14.9 \\
18.5\end{array}$ & $\begin{array}{l}<10^{-4} \\
\prime \prime \\
" \prime \\
\prime \prime \\
\prime \prime\end{array}$ & $\begin{array}{l}0.87 \\
4.08 \\
1.33 \\
4.26 \\
3.88\end{array}$ & $\begin{array}{l}10^{-2} \\
" 1 \\
\prime \prime \\
\prime \prime \\
\prime \prime\end{array}$ & $\begin{array}{l}3.22 \\
3.30 \\
3.46 \\
3.48 \\
4.75\end{array}$ & $\begin{array}{l}10^{-2} \\
11 \\
" 1 \\
" 1 \\
" 1\end{array}$ & $\begin{array}{l}1 \\
2 \\
3 \\
4 \\
5\end{array}$ & $\begin{array}{l}1 \\
1 \\
2 \\
1 \\
3\end{array}$ \\
\hline $\begin{array}{l}6 \\
7 \\
8 \\
9\end{array}$ & manila twine & $\begin{array}{l}42.1 \\
49.2 \\
79.2 \\
63.5\end{array}$ & $\begin{array}{l}\text { ", } \\
", \\
" \prime\end{array}$ & $\begin{array}{l}2.62 \\
2.61 \\
4.01 \\
3.15\end{array}$ & $\begin{array}{l}\text { ", } \\
\text { ", } \\
\text { "1 }\end{array}$ & $\begin{array}{l}16.1 \\
18.8 \\
19.7 \\
20.2\end{array}$ & $\begin{array}{l}\text { ", } \\
\text { ", } \\
\text { ", }\end{array}$ & $\begin{array}{l}1 \\
2 \\
3 \\
4\end{array}$ & $\begin{array}{l}1 \\
2 \\
1 \\
3\end{array}$ \\
\hline $\begin{array}{l}10 \\
11 \\
12 \\
13\end{array}$ & stras rope & $\begin{array}{r}27.9 \\
61.4 \\
121.5 \\
88.9\end{array}$ & $\begin{array}{l}\text { ", } \\
" 1, \\
11\end{array}$ & $\begin{array}{l}1.27 \\
2.74 \\
4.90 \\
3.72\end{array}$ & $\begin{array}{l}\text { "f } \\
\text { "f } \\
\text { ", }\end{array}$ & $\begin{array}{l}21.9 \\
22.4 \\
24.8 \\
23.9\end{array}$ & $\begin{array}{l}\text { ", } \\
\text { ", } \\
\text { "' }\end{array}$ & $\begin{array}{l}1 \\
2 \\
4 \\
3\end{array}$ & $\begin{array}{l}3 \\
4 \\
1 \\
2\end{array}$ \\
\hline $\begin{array}{l}14 \\
15 \\
16 \\
17\end{array}$ & Amilan & $\begin{array}{l}6.3 \\
3.5 \\
8.05 \\
10.2\end{array}$ & $\begin{array}{l}11 \\
11 \\
11 \\
\prime \prime\end{array}$ & $\begin{array}{l}3.08 \\
1.54 \\
3.30 \\
2.30\end{array}$ & $\begin{array}{l}11 \\
11 \\
" 1 \\
11\end{array}$ & $\begin{array}{l}2.05 \\
2.27 \\
2.44 \\
4.44\end{array}$ & $\begin{array}{l}\text { ", } \\
\text { ", } \\
\text { ", }\end{array}$ & $\begin{array}{l}1 \\
2 \\
3 \\
4\end{array}$ & $\begin{array}{l}1 \\
2 \\
4 \\
3\end{array}$ \\
\hline $\begin{array}{l}18 \\
19 \\
20 \\
21 \\
22 \\
23 \\
24\end{array}$ & Tevilon & $\begin{array}{l}28.5 \\
10.0 \\
21.0 \\
19.5 \\
32.0 \\
47.5 \\
75.5\end{array}$ & $\begin{array}{l}n \\
" 1 \\
n \\
" 1 \\
" 1 \\
" 1 \\
" 1\end{array}$ & $\begin{array}{l}4.96 \\
1.61 \\
2.57 \\
1.77 \\
2.32 \\
3.27 \\
4.44\end{array}$ & $\begin{array}{l}\text { "1 } \\
" 1 \\
" 1 \\
" 1 \\
1 " \\
1 " \\
1 "\end{array}$ & $\begin{array}{r}5.75 \\
6.21 \\
8.17 \\
11.0 \\
13.8 \\
14.5 \\
17.0\end{array}$ & $\begin{array}{l}\text { "t } \\
\text { "f } \\
\text { ", } \\
\text { ", } \\
\text { "1 } \\
\text { "! }\end{array}$ & $\begin{array}{l}1 \\
2 \\
3 \\
4 \\
5 \\
6 \\
7\end{array}$ & $\begin{array}{l}1 \\
3 \\
2 \\
4 \\
5 \\
7 \\
6\end{array}$ \\
\hline $\begin{array}{l}25 \\
26 \\
27 \\
28 \\
29 \\
30 \\
31\end{array}$ & silklon & $\begin{array}{r}4.1 \\
8.8 \\
17.6 \\
10.3 \\
16.2 \\
30.1 \\
36.6\end{array}$ & $\begin{array}{l}\text { "' } \\
" 1 \\
1 " \\
\text { ", } \\
11 \\
" \prime \\
" 1\end{array}$ & $\begin{array}{l}1.53 \\
2.74 \\
5.32 \\
2.21 \\
3.15 \\
5.45 \\
4.28\end{array}$ & $\begin{array}{l}\text { "1 } \\
11 \\
11 \\
" 1 \\
" 1 \\
1 " \\
11\end{array}$ & $\begin{array}{l}2.68 \\
3.21 \\
3.31 \\
4.66 \\
5.15 \\
5.52 \\
8.55\end{array}$ & $\begin{array}{l}\text { "1 } \\
\text { ", } \\
\text { ", } \\
\text { ", } \\
\text { ", }\end{array}$ & $\begin{array}{l}1 \\
2 \\
3 \\
4 \\
5 \\
6 \\
7\end{array}$ & $\begin{array}{l}1 \\
2 \\
3 \\
4 \\
4 \\
5 \\
6\end{array}$ \\
\hline $\begin{array}{l}32 \\
33 \\
34 \\
35 \\
36 \\
37\end{array}$ & Cremona & $\begin{array}{r}2.0 \\
13.4 \\
26.8 \\
12.5 \\
22.0 \\
26.5\end{array}$ & $\begin{array}{l}11 \\
11 \\
11 \\
1 ! \\
11 \\
1 "\end{array}$ & $\begin{array}{l}1.14 \\
4.43 \\
7.40 \\
1.95 \\
2.66 \\
2.89\end{array}$ & $\begin{array}{l}1 " \\
" 1 \\
" 1 \\
" 1 \\
" 1 \\
1 "\end{array}$ & $\begin{array}{l}1.75 \\
3.10 \\
3.62 \\
6.41 \\
8.27 \\
9.16\end{array}$ & $\begin{array}{l}\text { ", } \\
\text { ", } \\
\text { ", } \\
\text { ". }\end{array}$ & $\begin{array}{l}1 \\
2 \\
3 \\
4 \\
5 \\
6\end{array}$ & $\begin{array}{l}1 \\
2 \\
3 \\
4 \\
6 \\
5\end{array}$ \\
\hline $\begin{array}{l}38 \\
39 \\
40 \\
41 \\
42\end{array}$ & Kurehalon & $\begin{array}{l}55.4 \\
36.5 \\
73.0 \\
40.0 \\
80.5\end{array}$ & $\begin{array}{l}11 \\
11 \\
1 ! \\
y \\
n\end{array}$ & $\begin{array}{l}5.52 \\
2.40 \\
3.98 \\
1.93 \\
2.86\end{array}$ & $\begin{array}{l}\text { " } \\
\text { "f } \\
" 1 \\
" \prime \\
1 "\end{array}$ & $\begin{array}{l}10.1 \\
15.2 \\
18.3 \\
20.7 \\
28.2\end{array}$ & $\begin{array}{l}\text { "n } \\
" 1 \\
" t \\
" t \\
" 1\end{array}$ & $\begin{array}{l}1 \\
2 \\
3 \\
4 \\
5\end{array}$ & $\begin{array}{l}1 \\
2 \\
3 \\
4 \\
5\end{array}$ \\
\hline $\begin{array}{l}43 \\
44 \\
45 \\
46 \\
47\end{array}$ & Saran & $\begin{array}{r}53.3 \\
20.7 \\
68.3 \\
86.6 \\
111.2\end{array}$ & $\begin{array}{l}11 \\
11 \\
11 \\
11 \\
17\end{array}$ & $\begin{array}{l}5.37 \\
1.92 \\
2.18 \\
2.61 \\
2.66\end{array}$ & $\begin{array}{l}11 \\
11 \\
11 \\
11 \\
11\end{array}$ & $\begin{array}{c}9.92 \\
10.8 \\
31.3 \\
33.2 \\
41.8\end{array}$ & $\begin{array}{l}\text { "1 } \\
" 1 \\
1 " \\
" 1 \\
" 1\end{array}$ & $\begin{array}{l}1 \\
2 \\
3 \\
4 \\
5\end{array}$ & $\begin{array}{l}1 \\
2 \\
3 \\
4 \\
5\end{array}$ \\
\hline
\end{tabular}


(8)式应（7) 式比入礼る上

$$
\begin{aligned}
\tan \theta & =\frac{k m(2+\sigma m) l}{a \cdot v^{2}} \\
& =\frac{k d(2 l+\sigma d)}{a \cdot l \cdot v^{2}}
\end{aligned}
$$

從つて, $m, l, d, a, \rho, v$ の内, 佂意の 1 つが变つた㭙の $\theta$ の增減の㥞子は次式の正負の結果から求的 れる。

$$
\begin{aligned}
& \frac{\partial \theta}{\partial m}=\frac{2 k l}{a \cdot \nu^{2}}(1+\sigma m) \cos ^{2} \theta>0 \\
& \partial \theta=-\sigma k d^{2}-\cos ^{2} \theta<0 \\
& \frac{\partial \theta}{\partial d}=\frac{2 k(l+\sigma d)}{a \cdot l v^{2}}-\cos ^{2} \theta>0 \\
& \frac{\partial \theta}{\partial a}=\frac{-k d(2 l+\sigma d)}{a^{2} l v^{2}} \cos ^{2} \theta<0 \\
& \frac{\partial \theta}{\partial \rho}=\frac{\pi m(2+\sigma m l)}{4 a v^{2}} \cos ^{2} \theta>0 \\
& \frac{\partial \theta}{\partial v^{-}}=\frac{-2 k m(2+\sigma m) l}{a v^{3}} \cos ^{2} \theta<0
\end{aligned}
$$

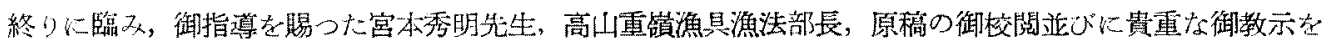

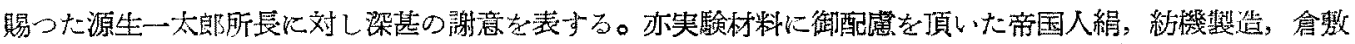

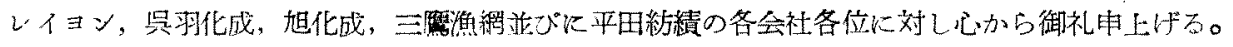

\section{女献}

1）野村正恒・野沢 靖：水打動く網地が受ける水の抵抗一N，網系の吹か九，本誌 $20(9) ， 762 \sim$ 769 (1955).

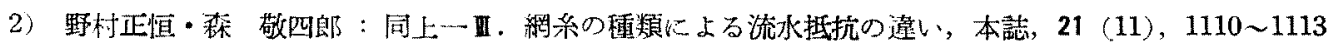
(1951).

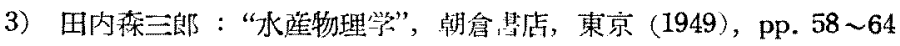

